\title{
Prévention des chutes
}

Olga Frank, René Schwendimann

1 Bureau suisse de prévention des accidents bpa. Les accidents en Suisse. Statistiques BPA 2007. Berne: Bubenberg; 2007.

2 Six P. Epidemiologie des Sturzes und der Hüftfraktur. Schweiz Rundschau Med (Praxis). 1992; 81(46):1378-82.

- Victorian Government Department of Human Services. Minimising the Risk of Falls \& Fall-related Injuries. Guidelines for Acute, Sub-acute and Residential Care Settings. 2004. www.health.vic.gov. au/qualitycouncil/pub/improve/ falls.htm. [Accès: 20.06.2007]

Correspondance:

Olga Frank

Diplômée en soins infirmiers

Fondation pour la Sécurité

des Patients

Asylstrasse 41

CH-8032 Zurich

Tél. 0432437672

Fax 0432437671

frank@patientensicherheit.ch

www.patientensicherheit.ch

\section{Introduction}

Chaque année, environ 80000 seniors de plus de 65 ans sont victimes d'accidents nécessitant des soins médicaux en Suisse. Les chutes (83\%) constituent le type d'accident le plus fréquent. Elles se soldent très souvent par une fracture du col du fémur [1]. Pour beaucoup de personnes âgées, la chute est l'événement le plus décisif et souvent le plus angoissant de leur vieillesse. Se retrouver brutalement par terre, entièrement démuni, est non seulement source de crainte et de douleur mais peut aussi perturber complètement le déroulement jusqu'alors positif de l'existence. Une chute peut déclencher un repli hors de la vie active, détruire la confiance en soi, anéantir toute perspective et être à l'origine d'une dépression [2]. Les chutes, et les lésions en résultant, sont souvent le signe d'un risque de perte de mobilité ou du début de cette perte. Ce problème sanitaire concerne en particulier les personnes âgées ou celles dont la santé est altérée par une maladie ou par des facteurs environnementaux inhabituels. C'est la raison pour laquelle les chutes sont particulièrement fréquentes dans les institutions sanitaires. Elles y sont un événement indésirable fréquent dans le cadre des traitements médicaux, infirmiers ou thérapeutiques. Chez ces personnes, il n'est pas rare que les chutes et leurs répercussions entraînent la nécessité d'une prise en charge définitive, voire même un décès. L'importance de cette problématique est bien connue des milieux professionnels de la santé. Cependant, les efforts entrepris pour l'introduction et l'application de directives systématiques internes, de nature préventive sont très inégaux.

C'est ce qui a incité la Fondation pour la Sécurité des Patients à éditer une publication sur la prévention des chutes à l'attention des hôpitaux, des établissements médico-sociaux, des services d'aide et de soins à domicile et des médecins traitants. L'objectif est de mettre un bilan des principaux aspects de la prévention des chutes, ainsi que des recommandations pratiques pour l'implémentation et la mise en œuvre de mesures multifactorielles ciblées à la disposition des établissements de santé. Cette publication fournit par ailleurs un support de décision aux professionnels et aux auxiliaires travaillant dans ces institutions

\section{Résumé}

Du fait de leur fréquence et de leurs répercussions physiques, psychiques et sociales, les chutes constituent un problème clinique majeur, de même qu'un risque sanitaire chez les personnes âgées. C'est pour ces raisons que la Fondation pour la Sécurité des Patients considère la problématique des chutes comme un champ d'action essentiel dans le cadre de la gestion du risque dans les établissements de santé, et qu'elle s'est consacrée à ce sujet en coopération avec l'Institut des sciences infirmières de l'Université de Bâle. Des recommandations concernant l'identification des chutes et des risques, ainsi que leur prévention ont été élaborées, en collaboration avec un groupe d'experts, pour les hôpitaux, les établissements de long séjour, les services d'aide et de soins à domicile et les médecins traitants. Les institutions de santé sont invitées à s'attaquer à ce problème et à implémenter des mesures adéquates dans le cadre de leur stratégie de qualité, ainsi qu'à promouvoir l'attention et l'expertise de leurs collaborateurs à un niveau interdisciplinaire.

- pour identifier les patients présentant des facteurs de risque de chute;

- pour éviter les chutes ou en réduire les suites par des interventions ciblées;

- pour protéger les patients qui sont déjà tombés contre de nouvelles chutes et leurs conséquences;

- pour promouvoir une documentation et une analyse systématiques dans ce domaine.

L'élaboration de cette publication «Prévention des chutes» se fonde sur l'étude de la littérature scientifique existant en la matière et sur l'expertise clinique d'un groupe de spécialistes interdisciplinaires et multiprofessionnels. Sa structure s'articule autour des étapes du processus de gestion du risque:

- $\quad 1^{\text {re }}$ étape: identification du risque;

- $\quad 2^{\mathrm{e}}$ étape: estimation du risque; 
- $\quad 3^{\mathrm{e}}$ étape: prévention et intervention;

- 4 e étape: évaluation;

- réaction aux chutes.

Cette publication informe - dans l'esprit d'un manuel pratique - des aspects pertinents qu'il convient de manière générale de prendre en compte pour l'identification et la prévention des chutes. Bien que les recommandations s'appliquent de la même manière aux hôpitaux, établissements de long séjour et aux institutions sanitaires de premier recours, les particularités y sont traitées à chaque étape en fonction du mandat spécifique de chacun de ces prestataires de services. La réaction aux chutes est par ailleurs traitée comme une étape distincte dans le cadre du processus de gestion des risques. Il s'agit d'une étape qui s'applique à toute chute et à tout moment, indépendamment de la chronologie du processus.

\section{$1^{\text {re }}$ étape: identification du risque}

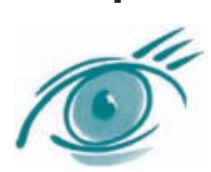

La première étape de la gestion du risque correspond à l'identification des patients présentant un risque accru. Cette publication fournit une liste des facteurs de risque ainsi que des instruments d'identification de celui-ci.

\section{$2^{\mathrm{e}}$ étape: estimation du risque}

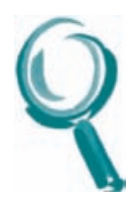

L'estimation des facteurs de risque (éventuels) d'un patient constitue un élément capital de la prévention des chutes. Elle a lieu dans le cadre d'une estimation (gériatrique) multidimensionnelle permettant la saisie et l'évaluation des problèmes et des ressources d'ordre médical, fonctionnel et psychosocial.

\section{$3^{\text {e }}$ étape: prévention et intervention}

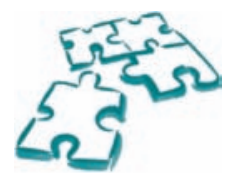

Cette étape englobe la planification et l'implémentation de mesures individuelles visant à réduire les chutes et à prévenir les lésions. Parallèlement à l'intégration du patient et de ses proches, la coordination interdisciplinaire de cette procédure est indispensable à l'efficacité des mesures de prévention. Dans ce contexte, le fait que les chutes et leurs répercussions constituent généralement un processus multifactoriel doit être pris en compte. Ceci implique que les mesures de prévention doivent être elles aussi à caractère multifactoriel et doivent être planifiées et mises en œuvre de manière systématique par une équipe interdisciplinaire.

\section{$4^{e}$ étape: évaluation}

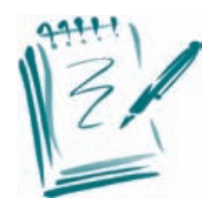

L'examen régulier des interventions prévues et réalisées fait partie du processus de gestion des risques. Lorsque l'état de santé et/ou l'environnement $\mathrm{du}$ patient et son traitement (notamment médicamenteux) changent ou que le patient fait une chute, une nouvelle évaluation doit avoir lieu afin de garantir une prévention adéquate et adaptée.

\section{Réaction aux chutes}

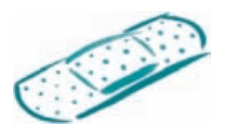

Après une chute, il convient de protéger la victime contre tout autre dommage. Après une évaluation clinique des conséquences et la prise de mesures d'urgence éventuelles, un procès-verbal structuré de cette chute en permettra une analyse systématique. Les données ainsi saisies serviront par ailleurs à l'assurance qualité (p.ex. statistiques des chutes/des lésions). Les conclusions tirées de cette analyse seront réintégrées en retour dans l'évaluation des facteurs de risque.

La publication «Prévention des chutes - Guide et recommandations à l'attention des hôpitaux, des établissements de long séjour et des institutions sanitaires de premier recours» met des connaissances spécifiques et des instruments à la disposition des médecins, des soignants et d'autres spécialistes et ce, sous une forme compacte. Lors de l'identification et de l'évaluation des risques de chute et de leurs causes, de la planification et de la réalisation des interventions requises, les spécialistes sont invités à coordonner leurs actions sur un plan interdisciplinaire et à veiller à une implémentation durable des mesures avec le soutien de la direction.

Cette publication est disponible en deux langues, allemand et français, auprès de la Fondation pour la Sécurité des Patients. Tous les instruments recommandés dans cette publication peuvent être par ailleurs téléchargés sur la page d'accueil de la Fondation (www. patientensicherheit.ch). Si un échange avec des institutions ayant déjà mis en œuvre des projets dans ce domaine et disposant donc d'une expérience en la matière est souhaité, la Fondation peut établir un contact avec des spécialistes de la prévention des chutes. 